\title{
Interaction between habitat quality and an Allee-like effect in metapopulations
}

\author{
R. McVinish ${ }^{a}$ and P.K. Pollett ${ }^{a}$

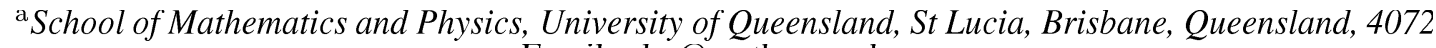 \\ Email:pkp@maths.uq.edu.au
}

\begin{abstract}
:
Many species exist as a collection of local populations occupying spatially distinct habitat patches. Such a collection of local populations is called a metapopulation. Metapopulations are constantly changing due to the processes of extinction and colonisation occurring at each habitat patch. The primary focus in the study of metapopulations is to determine if the metapopulation will persist and, if so, at what level.

Mathematical models of metapopulations provide ecologists with tools for better understanding the dynamics of the metapopulation. A useful class of metapopulation models is the stochastic patch occupancy models (SPOMs). The characterising feature of a SPOM is that only the presence/absence of a population at each habitat patch is modelled. Classical metapopulation models such as Levins's model and the stochastic logistic model assume homogeneity of habitat quality throughout the metapopulation. However, it is known that species distribution patterns are effected by the quality of habitat available [see Mortelliti et al., 2010, for an overview of the role of habitat quality]. Furthermore, it is necessary to incorporate habitat quality into the modelling in order to study the effect of habitat degradation and destruction on the persistence of the metapopulation. A review of some models that attempt to better reflect the ecological reality including incorporating variation in habitat quality is provided by Hanski and Ovaskainen [2003]. See also Gyllenberg and Hanski [1997] who study a differential equation metapopulation model that incorporates variation in habitat quality and use this model to examine the effect of habitat degradation and destruction.

In addition to habitat degradation, the persistence of a metapopulation is affected by its dynamical properties. One such property is called an Allee-like effect. This term is borrowed from population biology where the Allee effect refers to populations exhibiting a critical threshold below which the population goes extinct [see Courchamp et al., 2008, for detailed discussion of the Allee effect in ecology]. For metapopulations, an Allee-like effect refers to a metapopulation exhibiting a similar threshold behaviour. Amarasekare [1998] summarises some of the evidence supporting the operation of an Allee-like effect in real metapopulations and proposes a modification of the Levins's model which exhibits this phenomenon [see also Courchamp et al., 2008, pages 103-105]. Note that Amarasekare's model does not allow variation in habitat characteristics.

In this paper, we examine the effect of habitat degradation on a metapopulation exhibiting an Allee-like effect using the metapopulation model introduced in McVinish and Pollett [2010]. This model incorporates variation in habitat quality between patches by allowing the local survival probabilities to vary between patches (but not in time). The model can also incorporate an Allee-like effect by imposing certain conditions on the colonisation process. The resulting discrete time Markov chain is difficult to analyse directly, but can be approximated by a deterministic process when the number of habitat patches is large. This approximation is used to study the effect habitat degradation has on the persistence the metapopulation. We show that even a small amount of habitat degradation in metapopulations exhibiting an Allee-like effect can cause a metapopulation with a high level of persistence to go extinct. This can be contrasted with a metapopulation that does not exhibit an Allee-like effect where a small change to the habitat quality will result in only a small change to the level of persistence of the metapopulation. We conclude that metapopulations exhibiting an Allee-like effect are in much greater need of protection from habitat degradation and destruction.
\end{abstract}

Keywords: Allee effect, habitat destruction, metapopulation, SPOM. 


\section{A METAPOPUlation MOdel}

In this section, we summarise the model studied in McVinish and Pollett [2010]. This model is an example of a stochastic patch occupancy model (SPOM) as only the presence or absence of the species of interest is noted for each habitat patch and not the size or structure of the population at the habitat patch. Consider a metapopulation comprised of $n$ habitat patches and let $X_{t}^{(n)}$ indicate the state of this metapopulation at time $t$ where

$X_{t}^{(n)}=\left(X_{1, t}^{(n)}, \ldots, X_{n, t}^{(n)}\right) \quad$ with $\quad X_{i, t}^{(n)}= \begin{cases}1, & \text { if habitat patch } i \text { is occupied at time } t \\ 0, & \text { otherwise. }\end{cases}$

Between observations, the metapopulation undergoes a series of colonisation and extinction events. In this model, these events are separated into distinct phases as is done in Hill and Caswell [2001] and Buckley and Pollett [2010]. First, a colonisation phase occurs during which unoccupied patches are colonised by individuals from the occupied patches. The colonisation events are assumed to be independent of one another and the probability of an unoccupied patch being successfully colonised during this phase is given by a function, $f$, of the proportion of occupied patches in the metapopulation. The function $f$ is called the colonisation function and satisfies the following assumption;

(A) The colonisation function $f:[0,1] \mapsto[0,1]$ is an increasing, concave function such that $f(0)=0$ and $f^{\prime}(0)>0$.

The assumption that $f(0)=0$ means that once all habitat patches are unoccupied, they can not be recolonised from some outside source and the metapopulation is extinct. Concave colonisation functions such as $f(x)=1-\exp (-\beta x), \beta>0$ [Hill and Caswell, 2001] are commonly used, but non-concave functions are also relevant. The case of non-concave colonisation functions will he studied in the next section. Although this type of colonisation process ignores the connectivity and distances between habitat patches, it facilitates the analysis of the model. During the extinction phase, the local population at habitat patch $i$ goes extinct with probability $1-s_{i}$ independently of the other patches. We call $s_{i}$ the survival probability at patch $i$. Differences in survival probabilities between patches can arise simply due to differences in patch area [an assumption used, for example, in Moilanen, 2004], though other factors may have greater importance for population survival such as the abundance of food and shelter and the absence of predators.

Mathematically, the metapopulation model $\left\{X_{t}^{(n)}\right\}_{t=0}^{T}$ is a discrete time Markov chain. To describe the transitions, let $B(m, p)$ denote the binomial distribution with parameters $m \in\{0,1\}$ and $p \in[0,1]$. The transitions of $X_{t}^{(n)}$ are given by

$X_{i, t+1}^{(n)} \sim B\left(X_{i, t}^{(n)}, s_{i}\right)+B\left(1-X_{i, t}^{(n)}, s_{i} f\left(n^{-1} \sum_{j=1}^{n} X_{j, t}^{(n)}\right)\right)$.

The first term on the right hand side of (2) models a population occupying a habitat patch surviving the extinction phase, while the second term models an unoccupied patch being colonised and then the occupying population surviving the extinction phase. The complexity of the model prevents an exact treatment of the model. Instead we consider a deterministic approximation which is valid when the number of habitat patches is large. There is a large literature describing this type of approximation for continuous time Markov chains [see Darling and Norris, 2008, and references therein]. To construct the deterministic approximation of the metapopulation model, we impose the following assumption on the survival probabilities and initial state of the metapopulation.

(B) There exists a probability measure $\sigma$ and deterministic sequence $\{d(0, k)\}_{k=0}^{\infty}$ such that

$n^{-1} \sum_{i=1}^{n} s_{i}^{k} \stackrel{p}{\rightarrow} \bar{s}_{k}:=\int_{0}^{1} \lambda^{k} \sigma(d \lambda), \quad$ and $\quad n^{-1} \sum_{i=1}^{n} s_{i}^{k} X_{i, 0}^{(n)} \stackrel{p}{\rightarrow} d(0, k)$

for all $k=0,1, \ldots$ 
We call $\sigma$ the survival distribution. Assumption $(B)$ will hold if, for example, the $s_{i}$ are independent and identically distributed random variables with distribution $\sigma$ and if, given the $s_{i}$, the $X_{i, 0}^{(n)}$ are independent Bernoulli random variables with $P\left(X_{i, 0}^{(n)}=1 \mid s_{i}\right)=p\left(s_{i}\right)$ for some function $p$.

Theorem 1 (Theorem 2.1 of McVinish and Pollett [2010]) Suppose that $(B)$ holds. Then for all $k=$ $0,1, \ldots$ and all $t=0,1,2, \ldots$

$n^{-1} \sum_{i=1}^{n} s_{i}^{k} X_{i, t}^{(n)} \stackrel{p}{\rightarrow} d(t, k)$,

where

$d(t+1, k)=d(t, k+1)+f(d(t, 0))\left(\bar{s}_{k+1}-d(t, k+1)\right)$.

We are primarily interested in the sequence $\{d(t, 0)\}_{t=0}^{T^{\prime}}$ which gives the limiting proportion of occupied patches in the metapopulation. However, the other values of $d(t, k)$ do provide some useful information. For example, given $t$, the sequence $\{d(t, k) / d(t, 0)\}_{k=1}^{\infty}$ can be interpreted as the moments of the distribution of the survival probabilities of occupied patches.

To better understand the infinite system of difference equations (5), we examine its equilibrium points and their stability. A sequence $\{d(k)\}_{k=0}^{\infty}$ is an equilibrium point of (5) if it satisfies

$d(k)=d(k+1)+f(d(0))\left(\bar{s}_{k+1}-d(k+1)\right)$.

It is said to be stable if for all initial conditions $\{d(0, k)\}_{k=0}^{\infty}$ in a sufficiently small neighbourhood of $\{d(k)\}_{k=0}^{\infty}$

$\lim _{t \rightarrow \infty} d(t ; k)=d(k)$

for all $k \geq 0$. (Since, for each $t,\{d(t, k)\}_{k=0}^{\infty}$ is completely monotone, there exists a unique measure $\mu_{t}$ whose moments are given by $\{d(t, k)\}_{k=0}^{\infty}$ [see Theorem 4a in chapter III of Widder, 1941]. This is true also of the equilibrium points. A neighbourhood of the equilibrium point is interpreted as a weak neighbourhood of its associated measure. The limit (7) implies the weak convergence of $\mu_{t}$ to the measure associated with the equilibrium point.) Otherwise, the equilibrium point is called unstable. If the limit (7) holds for all initial conditions, then the equilibrium is said to be globally stable. The stable equilibrium point(s) of the system will provide an approximation of the long run proportion of occupied patches in the metapopulation. The following theorem gives the equilibrium points of (5) and their stability.

Theorem 2 Suppose that (A) holds. The equilibrium points of the recursion (5) are given by

$d(k)=\int_{0}^{1} \frac{f(\psi) \lambda^{k+1}}{1-\lambda+f(\psi) \lambda} \sigma(d \lambda)$,

where $\psi$ solves

$\psi=R_{\sigma}(\psi):=\int_{0}^{1} \frac{f(\psi) \lambda}{1-\lambda+f(\psi) \lambda} \sigma(d \lambda)$.

The extinction state, $d(k) \equiv 0$, is the unique equilibrium point of (5) and is globally stable if and only if

$f^{\prime}(0) \int_{0}^{1} \frac{\lambda}{1-\lambda} \sigma(d \lambda) \leq 1$

If inequality (10) does not hold, then the recursion (5) has two equilibrium points of which one is $d(k) \equiv$ 0 . The non-zero fixed point is stable, whilst $d(k) \equiv 0$ is unstable. 
Theorem 2 is mainly a restatement of Theorems 2.2 and 2.3 in McVinish and Pollett [2010]. Although it was not stated in McVinish and Pollett [2010] that $d(k) \equiv 0$ is globally stable if inequality (10) holds, this is evident from the proof. That $d(k) \equiv 0$ is unstable if inequality (10) does not hold is new and the proof is given in the appendix.

\section{INCORPORATING AN ALLEE-IIKE EFFECT}

An Allee-like effect for metapopulations can be defined as the metapopulation going extinct if the proportion of occupied patches is small, but otherwise the metapopulation has a positive long run proportion of occupied patches. In mathematical terms, this means that zero (the extinction state) is a stable equilibrium point of the system and that at least one non-zero, stable equilibrium point exists. In our model, from Theorem 2, as in the classical Levins's model and the limiting model of Buckley and Pollett [2010], if a non-zero stable equilibrium is present then zero is unstable. This implies the absence of Allee-like effects in the metapopulation.

Amarasekare [1998] proposed a modified version of Levins's model to study Allee-like effects in metapopulations. The model attempts to reproduce the phenomenon but model parameters do not keep the same interpretation. Hui and $\mathrm{Li}$ [2003] extended this model to incorporate both a 'rescue effect' and an 'overcrowding effect' while Zhou et al. [2004] considered a two species version of the model. Although each of these three papers consider the effect of habitat destruction, the assumption of homogeneous patch characteristics limited their analyses to considering either complete destruction of certain patches or a common reduction in the survival rates for all patches. Incorporating the Allee-like effect will permit a more detailed analysis of the interaction between habitat degradation and destruction and the Allee-like effect. To incorporate an Allee-like effect, we modify Assumption $(A)$.

$\left(A^{\prime}\right)$ The colonisation function $f:[0,1] \mapsto[0,1]$ is a non-concave, increasing function satisfying inequality (10) such that $f(0)=0$ and $f^{\prime \prime}(0)<0$.

Under assumption $\left(A^{\prime}\right)$, the fixed points of (5) are still given by equations (8) and (9). However, the number of solutions to (9) can not be easily determined. The assumption that inequality (10) is satisfied ensures that the extinction state is a stable equilibrium point of the system. The stability of other equilibrium points is determined by the following theorem.

Theorem 3 Let $\psi^{*}$ be a solution to (9). If $R_{\sigma}^{\prime}\left(\psi^{*}\right)<1$ then the corresponding equilibrium point given by equation (8) is stable. If $R_{\sigma}^{\prime}\left(\psi^{*}\right)>1$ then the corresponding equilibrium point is unstable.

The proof of Theorem 3 for $R_{\sigma}^{\prime}(\psi)<1$ follows the same arguments as in the proof of case (i) of Theorem 2.3 in McVinish and Pollett [2010]. Due to space constraints, the proof for $R_{\sigma}^{\prime}(\psi)>1$ is omitted but will appear elsewhere. In the modification of Levins's model, Amarasekare [1998] was able to explicitly determine a threshold for the initial metapopulation that determined to which of the two stable equilibrium points the metapopulation would approach. Due to the complexity of the model, we are unable to identify an explicit threshold. Instead, we derive a sufficient condition for the metapopulation to go extinct.

Theorem 4 Let $x^{*}$ be the smallest $x>0$ satisfying

$f(x) \int_{0}^{1} \frac{\lambda}{1-\lambda} \sigma(d \lambda)=x$.

If $\sum_{k=0}^{\infty} d(0 ; k)<x^{*}$ then $\lim _{t \rightarrow \infty} d(t ; 0) \rightarrow 0$.

The proof of Theorem 4 is given in the appendix. It is important to note that the condition in Theorem 4 concerns not only the proportion of occupied patches in the metapopulation but also the quality of those occupied patches. If the survival probabilities are bounded by $s^{*}$ then we can apply Theorem 4 to conclude that the metapopulation will go extinct if the initial proportion of occupied patches is less than $x^{*}\left(1-s^{*}\right)$. 


\section{Habitat DESTRUCTION AND DEgRAdATION}

Metapopulation models have often been used to study the effect of the habitat destruction on the behaviour of the metapopulation and, in particular, its effect on the equilibrium level. Most of these models were only able to reveal the effect of complete destruction of a certain proportion of the habitat patches [see, for example, Amarasekare, 1998; Hill and Caswell, 2001; Zhou et al., 2004]. One notable exception is the model studied by Gyllenberg and Hanski [1997] which incorporated patch quality and studied the interaction between habitat degradation and the rescue effect. In this paper, we consider a varying degree of habitat degradation at each habitat patch. Habitat degradation at patch $i$ is modelled by a decrease in the survival probability $s_{i} \mapsto s_{i}^{\prime}<s_{i}$. Complete destruction of patch $i$ is indicated by $s_{i} \mapsto 0$. The quality of the habitats in two metapopulations can be compared using the theory of stochastic ordering [Müller and Stoyan, 2002]. Let $\sigma_{1}$ and $\sigma_{2}$ be two distributions on $[0,1)$. We write $\sigma_{1} \leq \sigma_{2}$ if for all $x \in[0,1), \sigma_{1}((x, 1)) \leq \sigma_{2}((x, 1))$. We write $\sigma_{1}<\sigma_{2}$ if $\sigma_{1} \leq \sigma_{2}$ and if for some $x \in$ $[0,1), \sigma_{1}((x, 1))<\sigma_{2}((x, 1))$. The following two properties are important for our application: (i) If $\sigma_{1} \leq \sigma_{2}$, then for any increasing function $u, \int u(\lambda) \sigma_{1}(d \lambda) \leq \int u(\lambda) \sigma_{2}(d \lambda)$. (ii) If $\sigma_{1} \leq \sigma_{2}$ and $\int u(\lambda) \sigma_{1}(d \lambda)=\int u(\lambda) \sigma_{2}(d \lambda)$ for some strictly increasing function, then $\sigma_{1}=\sigma_{2}$.

First note that the integrand in equation (9) is increasing under both assumption $(A)$ and $\left(A^{\prime}\right)$. Therefore, if $\sigma_{1}<\sigma_{2}$ then for all $\psi \in[0,1], R_{\sigma_{1}}(\psi)<R_{\sigma_{2}}(\psi)$. Furthermore, we note that if $\sigma_{m}$ converges weakly to $\sigma$ then for all $\psi, R_{\sigma_{m}}(\psi) \rightarrow R_{\sigma}(\psi)$. In the absence of an Allee-like effect, that is under assumption $(A)$, these properties imply that the non-zero equilibrium point $\psi^{*}$ is a continuous, non-increasing function of $\sigma$. In other words, in the absence of an Allee-like effect, degrading the habitat will result in a gradual decrease of the equilibrium level. This point is illustrated in Figure 1 (Left).

The effect of habitat degradation can be more dramatic in the presence of an Allee-like effect. Suppose that $\sigma^{*}$ satisfies $R_{\sigma^{*}}(\psi) \leq \psi$ for all $\psi \in[0,1]$ and for some $\psi^{*}>0, R_{\sigma^{*}}\left(\psi^{*}\right)=\psi^{*}$. The only equilibrium point of a metapopulation with survival distribution $\sigma<\sigma^{*}$ is the extinction state $\psi^{*}=0$. On the other hand, a metapopulation with survival distribution $\sigma$ such that $\sigma^{*}<\sigma$ has at least one non-zero stable equilibrium point. This point is illustrated in Figure 1 (Right).

Finally, we note that equilibrium points with $R_{\sigma}^{\prime}\left(\psi^{*}\right)>1$ may increase under habitat degradation. However, Theorem 3 states that such equilibrium points are unstable and hence do not provide an approximation to the long run proportion of occupied patches in the metapopulation.

\section{Discussion}

Previous metapopulation models incorporating an Allee-like effect have been based on Levins's model Amarasekare [1998]; Zhou et al. [2004]. Therefore, they are unable to incorporate variation in habitat patch characteristics. We considered a variation of the metapopulation model of McVinish and Pollett [2010] which incorporates both the Allee-like effect and variation in habitat patch characteristics. This model was used to compare the effects of habitat degradation on metapopulations both with and without an Allee-like effect. It was demonstrated that for metapopulations with an Allee-like effect even a small amount of habitat degradation can have catastrophic consequences.

\section{APPENDix}

Proof of Theorem $2(d(k) \equiv 0$ is unstable if $(10)$ does not hold $)$ : If $d(k) \equiv 0$ were a stable fixed point then, $\lim _{t \rightarrow \infty} d(t ; 0)=0$ for $d(0 ; k)$ sufficiently small. Let $\phi_{t}(z)=\sum_{k=0}^{\infty} d(t ; k) z^{k}$ for all $z \in[0,1]$. Now if $d(t ; 0) \rightarrow 0$ then $\phi_{t}(z) \rightarrow 0$ for all $z \in(0,1)$ since $\phi_{t}(z)<d(t ; 0)(1-z)^{-1}$. We now show that, for some $z^{*} \in(0,1)$, it is not possible for $\phi_{t}\left(z^{*}\right) \rightarrow 0$. From recursion (5)

$$
\begin{aligned}
\phi_{t+1}(z)= & z^{-1} \phi_{t}(z)-z^{-1} d(t ; 0)+f(d(t ; 0))\left(\int_{0}^{1} \frac{\lambda}{1-\lambda z} \sigma(d \lambda)-z^{-1} \phi_{t}(z)+z^{-1} d(t ; 0)\right) \\
= & z^{-1} \phi_{t}(z)+d(t ; 0)\left(f^{\prime}(0) \int_{0}^{1} \frac{\lambda}{1-\lambda z} \sigma(d \lambda)-z^{-1}\right)-f(d(t ; 0)) z^{-1}\left(\phi_{t}(z)-d(t ; 0)\right) \\
& +\left(f(d(t ; 0))-f^{\prime}(0) d(t ; 0)\right) \int_{0}^{1} \frac{\lambda}{1-\lambda z} \sigma(d \lambda)
\end{aligned}
$$



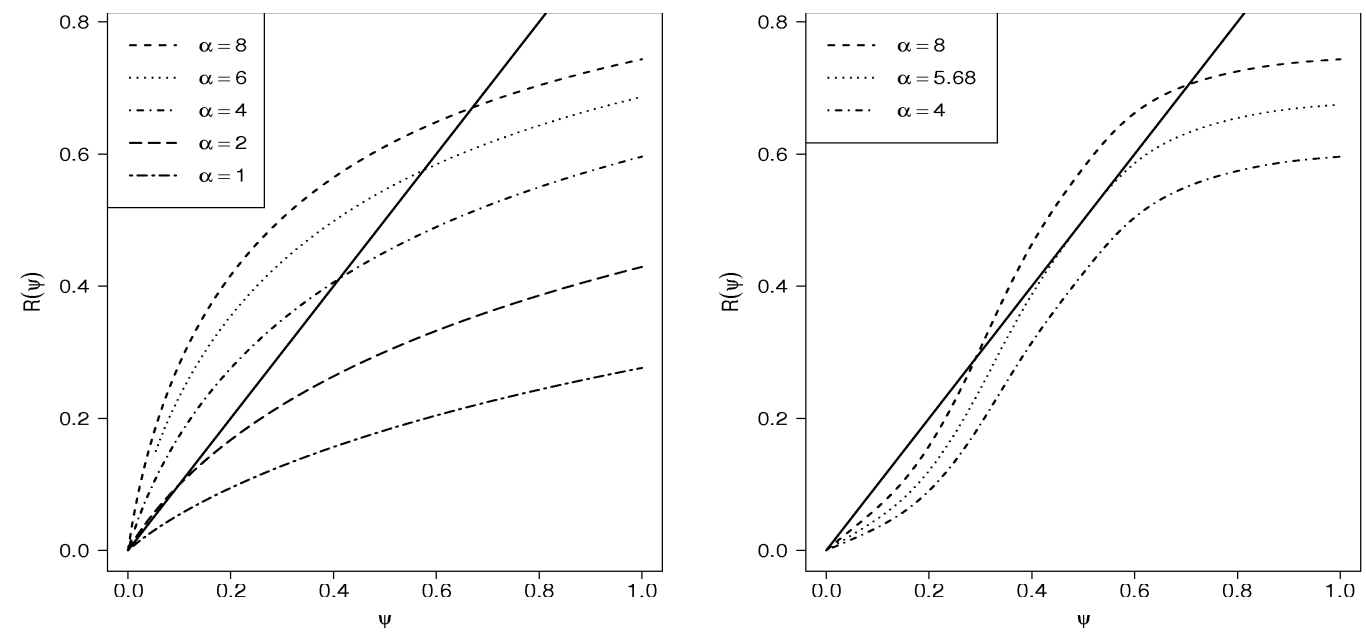

Figure 1: The functions $R_{\sigma}(\psi)$ are plotted for survival distributions $\operatorname{Beta}(\alpha, 2)$ with various values of $\alpha$. The intersection of the curve $R_{\sigma}(\psi)$ with the straight line gives the equilibrium levels of the metapopulations. Note that if $\alpha_{1}<\alpha_{2}$ then $\operatorname{Beta}\left(\alpha_{1}, 2\right)<\operatorname{Beta}\left(\alpha_{2}, 2\right)$ in the stochastic ordering. Left: No Allee-like effect. The colonisation function is $f(x)=0.7 x$ and $\alpha=8,6,4,2,1$. Right: Allee-like effect. A non-concave colonisation function is used with $f(1)=0.7$ and $\alpha=8,5.68,4$.

$$
\begin{aligned}
\geq & z^{-1}(1-f(d(t ; 0))) \phi_{t}(z)+d(t ; 0)\left(f^{\prime}(0) \int_{0}^{1} \frac{\lambda}{1-\lambda z} \sigma(d \lambda)-z^{-1}\right) \\
& +f^{\prime \prime}(\eta) d(t ; 0)^{2} \int_{0}^{1} \frac{\lambda}{1-\lambda z} \sigma(d \lambda),
\end{aligned}
$$

where $\eta \in[0, d(t ; 0)]$. Since inequality (10) does not hold, there exists a $\delta>0$ such that for all $z \in$ $\lceil 1-\delta, 1\rceil$,

$$
f^{\prime}(0) \int_{0}^{1} \frac{\lambda}{1-\lambda z} \sigma(d \lambda)-z^{-1} \geq 0 .
$$

As $f$ is concave, $f^{\prime \prime}(x) \leq 0$ for all $x \in[0,1]$. Let $C=-\min f^{\prime \prime}(x)$. If $\phi_{t}(z) \leq 1$ then for all $z \in[1-\delta, 1]$,

$$
\phi_{t+1}(z) \geq z^{-1}\left(1-f\left(\phi_{t}(z)\right)-C \phi_{t}(z) \int_{0}^{1} \frac{\lambda z}{1-\lambda z} \sigma(d \lambda)\right) \phi_{t}(z) .
$$

Therefore, there exists a $\rho>1$, an $\epsilon>0$ and $z^{*} \in(1-\delta, 1)$ such that if $\phi_{t}\left(z^{*}\right)<\epsilon$ then $\phi_{t+1}\left(z^{*}\right) \geq$ $\rho \phi_{t}\left(z^{*}\right)$. Hence, $\lim _{t \rightarrow \infty} \phi_{t}\left(z^{*}\right) \not \rightarrow 0$. This completes the proof.

Proof of Theorem 4: We first need to show that $\phi_{t}(1)<\infty$. If inequality (10) holds then

$$
\phi_{t}(1)=\sum_{k=0}^{\infty} d(t ; k) \leq \sum_{k=0}^{\infty} \bar{s}_{k}=1+\int_{0}^{1} \frac{\lambda}{1-\lambda} \sigma(d \lambda)<\infty .
$$

Noting that $d(t ; 0)<\phi_{t}(1)$, recursion (5) gives

$$
\phi_{t+1}(1)<\phi_{t}(1)+d(t ; 0)\left(\frac{f(d(t ; 0))}{d(t ; 0)} \int_{0}^{1} \frac{\lambda}{1-\lambda} \sigma(d \lambda)-1\right) .
$$

Set $x^{*}$ to be the smallest $x>0$ satisfying equation (11). If $\phi_{t}(1) \leq x^{*}$ then $d(t ; 0) \leq x^{*}$ and $\phi_{t+1}(1)<$ $\phi_{t}(1)$. This establishes that $\phi_{t}(1)$ is a decreasing sequence. Using the same arguments as in the proof 
of case (ii) of Theorem 2.3 in McVinish and Pollett [2010], we establish that $\lim _{t \rightarrow \infty} d(t, k)=0$ for all $k \geq 1$. We rewrite recursion (5) as

$$
d(t+1 ; 0) \leq \delta_{t}+\frac{f(d(t ; 0))}{d(t ; 0)} \bar{s}_{1} d(t ; 0),
$$

where $\delta_{t}$ is a positive, bounded sequence converging to zero. For all $x<x^{*}$,

$$
\frac{f(x)}{x} \bar{s}_{1} \leq \frac{f(x)}{x} \int_{0}^{1} \frac{\lambda}{1-\lambda} \sigma(d \lambda)<1
$$

from equation (11) and using the continuity of $f$. It now follows that $\lim _{t \rightarrow \infty} d(t, 0)=0$. This completes the proof.

\section{ACKNOWLEDGMENTS}

This work is supported by the Australian Research Council (Discovery grant DP110101929 and Centre of Excellence for Mathematics and Statistics of Complex Systems).

\section{REFERENCES}

Amarasekare, P. (1998) Allee effects in metapopulation dynamics, The American Naturalist, 152, 298302.

Buckley, F.M. and Pollett, P.K. (2010) Limit theorems for discrete-time metapopulation models. Probability Surveys, 7, 53-83.

Courchamp, F., Berec, L. and Gascoigne, J. (2008) Allee effects in ecology and conservation, Oxford University Press.

Darling, R.W.R. and Norris, J.R. (2008) Differential equation approximations for Markov chains, Probability Surveys, 5, 37-79.

Gyllenberg, M. and Hanski, I. (1997) Habitat deterioration, habitat destruction, and metapopulation persistence in a heterogeneous landscape. Theoretical Population Biology, 52, 198-215.

Hanski, I. and Ovaskainen, O. (2003) Metapopulation theory for fragmented landscapes. Theoretical Population Biology, 64, 119-127.

Hill, M.F. and Caswell, H. (2001) The effects of habitat destruction in finite landscapes: A chain-binomial metapopulation model. Oikos, 93, 321-331.

Hui, C. and Li, Z. (2003) Dynamical complexity and metapopulation persistence. Ecological Modelling, 164, 201-209.

Moilanen, A. (2004) SPOMSIM: software for stochastic patch occupancy models of metapopulation dynamics. Ecological Modelling, 179, 533-550.

McVinish, R. and Pollett, P.K. (2010) Limits of large metapopulations with patch-dependent extinction probabilities, Advances in Applied Probability, 42, 1172-1186.

Mortelitti, A., Amori, G. and Boitani, L. (2010) The role of habitat quality in fragmented landscapes: a conceptual overview and prospectus for future research. Oecologia, 163, 535-547.

Müller, A. and Stoyan, D. (2003) Comparison methods for stochastic models and risks, Wiley.

Widder, D.V. (1941) The Laplace Transform, Princeton University Press.

Zhou, S.-R., Lui, C.-Z. and Wang, G. (2004) The competitive dynamics of metapopulation subject to the Allee-like effect. Theoretical Population Biology, 65, 29-37. 